Pacific Journal of Mathematics

ON EMBEDDING A COMPACT CONVEX SET INTO A
SCALY CONVEX TOPOLOGICAL VECTOR SPACE

ROBERT EDWARD JAMISON, II, RICHARD O'BRIEN
AND PETER DRUMMOND TAYLOR 


\title{
ON EMBEDDING A COMPACT CONVEX SET INTO A LOCALLY CONVEX TOPOLOGICAL VECTOR SPACE
}

\author{
R. E. JAMISON, R. C. O'BRIEN AND P. D. TAYLOR
}

If a compact convex subset of a topological vector space is strongly locally convex then it is affinely homeomorphic to a convex subset of a locally convex topological vector space.

Throughout this paper, a convex set $X$ will be a convex subset of a real Hausdorff topological vector space endowed with the relative topology. If the relative topology is (locally) compact we will say that $X$ is a (locally) compact convex set.

Usually, when one proves results about compact convex sets, for example in the Krein-Milman and Ryll-Nardzewski Theorems, one requires that the containing vector space be locally convex. It is not known whether this requirement is necessary. It would not be necessary, for example, if every compact convex set could be embedded by an affine homeomorphism into a locally convex topological vector space. But it is not known whether such embeddings always exist. ${ }^{1}$ In this paper we show that if a compact convex set satisfies a certain local convexity condition, then it can be so embedded.

A convex set $X$ is strongly locally convex if, for each $x$ in $X$ and neighborhood $U$ of $x$ contained in $X$, there is an open convex neighborhood of $x$ contained in $U$. In other words, $X$ is strongly locally convex if its topology has a base of open convex sets. A convex subset of a locally convex topological vector space is evidently strongly locally convex. The principal result of this paper is the converse statement above.

An extension of this to the locally compact case is also given.

In $\$ 2$ we show that the real-valued lower semicontinuous affine functions on a convex set always separate disjoint open convex sets. If $X$ is a compact convex set which is strongly locally convex, then we show in $\$ 3$ that a "barycentre map" can be defined for $X$. This allows us to prove the main theorem which we extend to locally compact topologies in $\S 4$. In $\$ 5$ we give some open questions and partial results.

2. Affine functions on convex sets. We now commence

${ }^{1}$ Note Added in Proof. An example has been constructed by James W. Roberts of a compact convex set with no extreme points, which cannot therefore be embedded in a locally convex TVS. 
the step leading to the proof of the main theorem with an investigation of affine functions on a convex set $X$. For any affine function $f: X \rightarrow R$, let

$$
Z(f)=\{x \in X: f(x)=0\} .
$$

Proposition 1. Let $X$ be a convex set. If $f: X \rightarrow R$ is an affine function such that (i) for some two points $p_{0}$ and $q_{0}$ of $X, f\left(p_{0}\right)<0$ and $f\left(q_{0}\right)>0$, and (ii) for each $x$ in $\operatorname{cl} Z(f), f(x) \leqq 0$, then $f$ is lower semicontinuous on $X$.

Proof. As $f$ may be altered by any positive scalar multiple without changing the sense of the proposition, we may suppose that $f\left(p_{0}\right)<-1$ and $f\left(q_{0}\right)>1$. Thus we may select on the line segment from $p_{0}$ to $q_{0}$ two points $p$ and $q$ with $f(p)=-1$ and $f(q)=1$.

We are required to show that, for each $r$ in $R, L_{r}=\{x \in X: f(x) \leqq$ $r\}$ is closed in $X$. If $x$ is a limit point of $L_{r}$, there is a net $\left\{x_{\gamma}\right\}$ of points in $X$ converging to $x$ with $f\left(x_{\gamma}\right) \leqq r$ for each $\gamma$. By passing to subnets, if necessary, we may break the argument into two cases: (1) $f\left(x_{\gamma}\right)>0$ for all $\gamma$, or (2) $f\left(x_{\gamma}\right) \leqq 0$ for all $\gamma$. Passing to subnets again, we further obtain two ancillary cases: (a) $f\left(x_{\gamma}\right)$ converges in $R$ to some value, say $s$, with $s \leqq r$, or (b) $f\left(x_{\gamma}\right) \rightarrow-\infty$.

In case (1), the subcase (a) must obtain since the net $\left\{f\left(x_{\gamma}\right)\right\}$ is bounded below by 0 . In this situation define for each $\gamma, y_{\gamma}=$ $\left(1+f\left(x_{\gamma}\right)\right)^{-1}\left(x_{\gamma}+f\left(x_{\gamma}\right) p\right)$, a convex combination of $x_{\gamma}$ and $p$ such that $f\left(y_{\gamma}\right)=0$. By continuity of convex combination, $y_{\gamma}$ converges to $(1+s)^{-1}(x+s p)$. Since $y_{\gamma} \in Z(f)$, hypothesis (ii) guarantees that $f\left([1+s]^{-1}(x+s p)\right) \leqq 0$. Since $f$ is affine, this yields $f(x) \leqq-s f(p)=s \leqq$ $r$, as desired.

In case (2), set $y_{\gamma}=\left(1-f\left(x_{\gamma}\right)\right)^{-1}\left(x_{\gamma}-f\left(x_{\gamma}\right) q\right)$ for each $\gamma$. Then $y_{\gamma}$ is a convex combination of $x_{\gamma}$ and $q$ such that $f\left(y_{\gamma}\right)=0$. If (b) obtains, the coefficient $\left[1-f\left(x_{\gamma}\right)\right]^{-1}$ of $x_{\gamma}$ in the definition of $y_{\gamma}$ tends to 0 as $f\left(x_{\gamma}\right)$ goes to $-\infty$. Thus $y_{\gamma} \rightarrow 0 \cdot x+1 \cdot q=q$. This is untenable, as it would force $q \in \operatorname{cl} Z(f)$ and hence $f(q) \leqq 0$, contrary to the choice of $q$. Thus (a) must again hold, so $\left\{y_{\gamma}\right\}$ converges to $(1-s)^{-1}(x-s q)$. (Note that $s \leqq 0$ since $f\left(x_{\gamma}\right) \leqq 0$ for all $\gamma$.) Using the hypothesis on $\operatorname{cl} Z(f)$ once more, we get

$$
f\left([1-s]^{-1}(x-s q)\right) \leqq 0 \quad \text { whence. } \quad f(x) \leqq s f(q)=s \leqq r .
$$

Therefore, in this case as well, $x \in L_{r}$, so $L_{r}$ is closed.

Propósition 2. If $X$ is a compact convex set, then every lower semicontinuous affine function on $X$ is bounded both from below and above on $X$. 
Proof. A lower bound follows immediately from routine considerations. To establish an upper bound, let us suppose in contradiction that $f$ is a lower semicontinuous affine function on $X$ which is not bounded from above. Then $X$ is the countable union of the closed sets $\{x \in$ $X: f(x) \leqq n\}$. Since $X$ is compact, it is of the second Baire category in itself [2, p. 201]. Hence, for some $N>1,\{x: f(x) \leqq N\}$ has nonempty interior. Choose a point $p$ in the interior. Since by supposition $f$ is not bounded above, we can pick, for each $n>N$, some $x_{n}$ in $X$ with $f\left(x_{n}\right)>n$. Letting $\lambda_{n}=(n-N) /(n-f(p))$, set $y_{n}=\lambda_{n} p+$ $\left(1-\lambda_{n}\right) x_{n}$. Then

$$
f\left(y_{n}\right)>\lambda_{n} f(p)+\left(1-\lambda_{n}\right) n=n-\lambda_{n}(n-f(p))=N .
$$

Since $f(p)$ and $N$ are fixed numbers, $\lambda_{n} \rightarrow 1$ as $n \rightarrow \infty$. The compactness of $X$ allows us to find a convergent subnet $x_{n(\gamma)} \rightarrow x$ whence $\left\{y_{n(\gamma)}\right\}$ converges to $1 \cdot p+0 \cdot x=p$. But $f\left(y_{n(\gamma)}\right)>N$ for each $\gamma$, contrary to the choice of $p$ in the interior of $\{x \in X: f(x) \leqq N\}$.

Proposition 3. Suppose that $X$ is a convex set and that $K$ is a closed convex subset of $X$. If $K$ has nonempty interior and $X \sim K$ is nonempty and convex, then there is an affine function $f$ on $X$ such that $\{x \in$ $X: f(x) \leqq 0\}=K$.

Proof. Since the result is obvious if the linear space $E$ containing $X$ is one dimensional, we shall assume that the dimension of $E$ is at least 2. Let $p$ be any fixed point in the interior of $K$. For any point $u$ of $U=X \sim K$, define $g(u)=\sup \{\lambda: \lambda u+(1-\lambda) p \in K\}$. Since $p$ is interior to $K$, it follows that $g(u)>0$. Since $K$ is closed, $g(u)<1$. Thus setting $f^{\prime}(u)=(1-g(u)) / g(u)$, we have $f^{\prime}(u)>0$ for all $u$ in $U$.

We claim that $f^{\prime}$ is affine on $U$. To see this, suppose $v$ and $w$ are points in $U$ and $\beta \in[0: 1]$. Let $z=\beta v+(1-\beta) w$, and let $F$ be a 2-dimensional flat containing $v, w$ and $p$. The convex slice $X \cap F$ is the union of two disjoint convex subsets $K \cap F$ and $U \cap F$. It follows that the common boundary (in the usual sense) of $K \cap F$ and $U \cap F$ is the intersection of $X \cap F$ with some line $L$ in $F$. Since $p$ is interior to $K$ and hence not linearly accessible from $U, p$ does not lie on $L$. Therefore there is a unique affine function $h$ on $F$ which vanishes on $L$ and is -1 at $p$. For any point $u$ in $U \cap F$, the definition of $g$ implies that $r=g(u) u+(1-g(u)) p$ belongs to the bounding line $L$, so

$$
0=h(r)=g(u) h(u)+(1-g(u)) h(p) .
$$

Using $h(p)=-1$ and solving yields $h(u)=f^{\prime}(u)$ for any $u$ in $U \cap F$. Since $v, w$ and $z$ all belong to $U \cap F$, 


$$
f^{\prime}(z)=h(z)=\beta h(v)+(1-\beta) h(w)=\beta f^{\prime}(v)+(1-\beta) f^{\prime}(w) .
$$

It follows that $f$ is affine on $U$.

We now wish to extend the domain of definition of $f^{\prime}$ to all of $X$. Since $f^{\prime}$ is an affine function on the convex subset $U$ of $E$, there is some (purely algebraic) affine extension of $f^{\prime}$ to all of $E$. By choosing some such extension and restricting it to $X$, we obtain an affine function $f$ on $X$ which agrees with $f^{\prime}$ on $U$.

It remains to show that $\{x \in X: f(x) \leqq 0\}=K$. As already noted at the definition of $f^{\prime}, f(u)=f^{\prime}(u)>0$ for all $u$ in $U=X \sim K$. Thus we need to show now that if $x \in K$, then $f(x) \leqq 0$. To do so, consider any point $u$ of $U$ and set $\epsilon=f^{\prime}(u)=f(u)>0$. Solving for $g(u)$ in the definition of $f^{\prime}$, we get $g(u)=(1+\epsilon)^{-1}$, so that the point $q=$ $(1+\epsilon)^{-1} u+[\epsilon /(1+\epsilon)] p$ is the last point of $K$ on the segment from $p$ to $u$. Now for any point $u^{\prime}=\lambda u+(1-\lambda) q$, where $\lambda \in(0,1]$, it is a straightforward albeit tedious computation to check that, by definition of $f^{\prime}, f^{\prime}\left(u^{\prime}\right)=\lambda \epsilon=\lambda f(u)$. Because $f$ is affine on $X$, it can be deduced from this that $f(q)=0$ and $f(p)=-1$.

It is an easy conclusion from the above discussion that if $x \in K$ is colinear with $p$ and a point of $U$, then $f(x) \leqq 0$. Thus suppose $x$ does not lie on a line through $p$ and a point of $U$, and assume, contrary to our desire, that $f(x)>0$. Since $f(p)=-1$, there is a unique point $y$ on the line segment from $p$ to $x$ with $f(y)=0$. Select a point $u$ of $U$, and let $w$ be a point other than $u$ in the intersection of $U$ with the segment from $x$ to $u$. Choose $q$ as above on the segment from $p$ to $u$. The line segment from $w$ to $p$ is then cut at a point $x^{\prime}$ by the segment from $x$ to $q$ and at a point $y^{\prime}$ by the segment from $y$ to $q$. Since $x \neq y$, it follows that $x^{\prime} \neq y^{\prime}$.

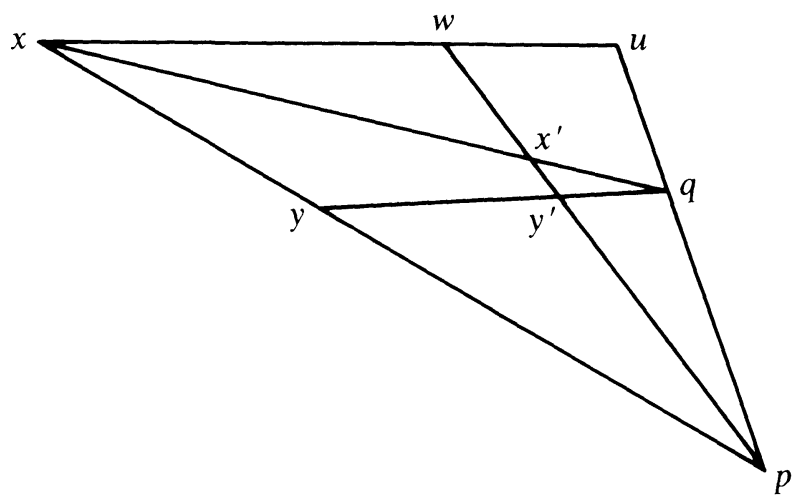

Since $f(y)=f(q)=0, f\left(y^{\prime}\right)=0$. Clearly $y^{\prime}$ lies between $x^{\prime}$ and $p$, so $f\left(x^{\prime}\right)>0$. But $x^{\prime}$ lies in $K$ since $x$ and $q$ are in $K$ and $K$ is convex. Thus $f\left(x^{\prime}\right) \leqq 0$ since it is colinear with $p$ and the point $w$ of 
$U$. Because of this contradiction, we are forced to conclude that $f(x) \leqq 0$, and the proposition is proved.

Proposition 4. Let $X$ be a convex set. If $U$ and $V$ are disjoint nonempty convex open sets in $X$, then there is a lower semicontinuous affine function $f: X \rightarrow \mathbf{R}$ such that $f(x)<0$ for all $x$ in $U$ and $f(y)>0$ for all $y$ in $V$.

Proof. By Zorn's lemma, there is a maximal convex subset $K$ of $X$ with $U \subseteq K$ and $V \cap K=\varnothing$. The closure $\bar{K}$ of $K$ is again convex. Since $V$ is open, $\bar{K} \cap V=\varnothing$, so by maximality of $K$, it follows that $K=\bar{K}$. As $V$ is convex, the maximality of $K$ in $X \sim V$ implies that $X \sim K$ is convex [3, p. 17]. Thus from Proposition 3, there is an affine function $f: X \rightarrow \mathbf{R}$ with $K=\{x \in X: f(x) \leqq 0\}$. Because $K$ is closed, Proposition 1 implies that $f$ is lower semicontinuous. Since $V \cap K=\varnothing$, $f(y)>0$ for all $y$ in $V$. Because $U$ is open and included in $K$, it follows readily that $f(x)<0$ for all $x$ in $U$.

If at this point we could demonstrate the full continuity of the affine function produced above, the main theorem would follow immediately (since then the natural embedding of $X$ into the dual space of the real-valued affine continuous functions on $X$ with the weak ${ }^{*}$ topology would be a continuous affine injection). Unfortunately, as the next example shows, this affine function may fail to be continuous.

Example A. Let $X$ denote the set of all real-valued sequences $\left(x_{n}\right)_{n=0}^{\mathrm{x}}$ such that

(i) $\quad-2 \leqq x_{0} \leqq 0$,

(ii) $0 \leqq x_{n}$ for $n \geqq 1$, and

(iii) $\sum_{n=1}^{x} x_{n} \leqq 1$.

Then $X$ is a convex subset of $l_{1}$ which is compact in the topology of co-ordinatewise convergence and the function $f:\left(x_{n}\right)_{n=0}^{\infty} \rightarrow \sum_{n=0}^{x} x_{n}$ is a lower semicontinuous affine function on $X$. Let $K=\{x \in X: f(x) \leqq 0\}$. Any sequence $\left(x_{n}\right)_{n=0}^{\infty}$ with $-2 \leqq x_{0}<-1$ is in the interior of $K$ and $X \sim K$ is nonvoid and convex. The function $f$ (or some positive multiple of it) is the function which Proposition 3 would associate with $K$, but $f$ is not continuous on $X$.

3. The Barycentre map. Throughout this section $X$ will be a compact convex set. $M(X)$ will denote the space of Radon measures on $X$ with the weak* topology (from $C(X)$ ) and $P(X)$ the compact convex subset of probability measures on $X$. Let $A(X)$ be the set of real-valued affine continuous functions on $X$, and $L(X)$ be the set of real-valued affine lower semicontinuous functions on $X$. 
A barycentre map for $X$ is an affine continuous map $r: P(X) \rightarrow X$ such that $r\left(\delta_{x}\right)=x$. $\quad\left(\delta_{x}\right.$ denotes the unit mass at $x$. $)$ Let us call a measure discrete if it lives on a finite set of points. Then a barycentre map is uniquely determined on discrete measures: $r\left(\sum \alpha_{l} \delta_{x_{t}}\right)=\Sigma \alpha_{i} x_{l}$. Since the discrete measures are weak ${ }^{*}$ dense, and $r$ is required to be continuous, there is at most one barycentre map on $X$. If $A(X)$ separates points of $X$, one can use $A(X)$ to define a barycentre map for $X$; indeed this is how we obtain the usual resultant map [6, p. 2]. The main tool of this section is the result that if we can define a barycentre map on $X$, then $A(X)$ must separate points.

The following proposition gives us the technical result we need to define a barycentre map. If $\mu=\Sigma \alpha_{i} \delta_{x_{i}}$ is a discrete probability measure on $X$, the resultant of $\mu$ is $\Sigma \alpha_{1} x_{i}$.

Proposition 5. Suppose that $X$ is strongly locally convex. Let $\left\{\mu_{\alpha}\right\}$ be a net of discrete probability measures on $X$ converging to $\mu$ in $P(X)$. If $x_{\alpha}$ is the resultant of $\mu_{\alpha}$, then the net $\left\{x_{\alpha}\right\}$ converges to some $x \in X$.

Proof. The following lemma gives us a candidate for $x$.

Lemma. For any $\mu \in P(X)$, there exists $x \in X$ such that $f(x) \leqq$ $\mu(f)$ for every $f \in L(X)$.

Proof. Suppose $\mu \in P(X)$ is fixed. By lower semicontinuity $\{x: \mu(f) \geqq f(x)\}$ is closed for each $f \in L(X)$. Hence by compactness we need only show that given $f_{1}, \cdots, f_{n} \in L(X)$, we have $\bigcap_{t=1}^{n}\left\{x: \mu\left(f_{t}\right) \geqq\right.$ $\left.f_{i}(x)\right\} \neq \varnothing$.

So suppose $f_{1}, \cdots, f_{n}$ in $L(X)$ are fixed. Define $K \subset \mathbf{R}^{n}$ by

$$
\begin{gathered}
K=\left\{\left(y_{1}, \cdots, y_{n}\right): \text { for some } x \in X \text { and all } i=1, \cdots, n,\right. \\
\left.y_{\imath} \geqq f_{i}(x)\right\} .
\end{gathered}
$$

Since the $f_{1}$ 's are affine, it follows easily that $K$ is convex. Moreover, we claim $K$ is closed. For suppose $\xi^{m}=\left(\xi_{1}^{m}, \cdots, \xi_{n}^{m}\right) \in K$ and $\xi^{m} \rightarrow \xi=$ $\left(\xi_{1}, \cdots, \xi_{n}\right)$. Then for each $m$, there exists $x_{m} \in X$ such that $f_{i}\left(x_{m}\right) \leqq \xi_{i}^{m}$ for all $i$. Let $x_{\alpha} \rightarrow x$ be a convergent subnet of $\left\{x_{m}\right\}$. For all $i$, by lower semicontinuity, $\lim f_{i}\left(x_{\alpha}\right) \geqq f_{i}(x)$, and thus $f_{i}(x) \leqq \lim _{m \rightarrow \infty} \xi_{\imath}^{m}$, implying that $f_{i}(x) \leqq \xi_{1}$. So $\xi \in K$ and $K$ is closed.

Now write $\xi=\left(\mu\left(f_{1}\right), \cdots, \mu\left(f_{n}\right)\right)$. By Proposition $2, \mu\left(f_{i}\right)$ is finite for every $i$. To prove the lemma, we must show $\xi \in K$.

If $\xi \notin K$, then since $K$ is closed and convex there is on $\mathbf{R}^{n}$ a linear functional $T:\left(y_{1}, \cdots, y_{n}\right) \rightarrow \sum_{i=1}^{n} \alpha_{\imath} y_{1}$ which strictly separates $K$ and $\xi$. That is, $T(x)>T(\xi)$ for each $x$ in $K$. Since $T$ is bounded below on 
$K$, it is clear from the definition of $K$ that no $\alpha_{\imath}$ can be negative. Thus $\alpha_{\imath} \geqq 0$ for all $i$ so that the linear function $g=\sum_{i=1}^{n} \alpha_{i} f_{i}$ belongs to $L(X)$.

Choose $y \in X$ such that $\mu(g) \geqq g(y)$. (Any $y$ at which $g$ achieves its infimum will do.) Then

$$
\begin{aligned}
T(\xi) & =\sum_{i=1}^{n} \alpha_{\imath} \mu\left(f_{\imath}\right)=\mu\left[\sum_{\imath=1}^{n} \alpha_{\imath} f_{l}\right]=\mu(g) \geqq g(y) \\
& =\sum_{i=1}^{n} \alpha_{\imath} f_{\imath}(y)=T\left(f_{1}(y), f_{2}(y), \cdots, f_{n}(y)\right) \\
& \geqq \inf _{k \in K} T(k)>T(\xi) .
\end{aligned}
$$

This contradiction proves the lemma.

We turn now to the proof of the proposition.

The lemma gives us an $x \in X$ such that $f(x) \leqq \mu(f)$ for every $f \in L(X)$. It is well-known (and easy to show) that for any $f \in L(X)$, the map $\mu \rightarrow \mu(f)$ is lower semicontinuous on $P(X)$. So for every $f \in L(X)$, we have:

$$
\underline{\lim } f\left(x_{\alpha}\right)=\underline{\lim } \mu_{\alpha}(f) \geqq \mu(f) \geqq f(x) .
$$

If $\left\{x_{\alpha}\right\}$ does not converge to $x$, then there exists a subnet $\left\{x_{\beta}\right\}$ which converges to a point $y \in X, y \neq x$. Since $X$ is strongly locally convex, by Proposition 4 there exists a neighborhood $U$ of $y$ and $f \in L(X)$ such that $f(U)<0<f(x)$. But then for this particular $f$, we have

$$
\underline{\lim } f\left(x_{\alpha}\right) \leqq \underline{\lim } f\left(x_{\beta}\right) \leqq 0<f(x) .
$$

This contradiction implies $\left\{x_{\alpha}\right\} \rightarrow x$.

COROllary. If $X$ is strongly locally convex, for any $\mu \in P(X)$ there is a unique $x \in X$ such that for any net $\left\{\mu_{\alpha}\right\}$ of discrete probability measures converging to $\mu$, the net $\left\{x_{\alpha}\right\}$ of resultants converges to $x$.

Proposition 6. Suppose that $X$ is strongly locally convex. Then there is a barycentre map for $X$.

Proof. For any $\mu \in P(X)$, define $r(\mu)$ to be the unique $x \in X$ given by the Corollary to Proposition 5 .

Clearly $r\left(\delta_{x}\right)=x$ and it is easy to show that $r$ is affine.

To prove that $r$ is continuous suppose $\left\{\mu_{\alpha}\right\}$ is a net converging weak* to $\mu$. Let $\beta$ index a weak* neighborhood basis $N_{\beta}$ of 0 in $M(X)$. For 
each $\alpha$ and $\beta$ choose a discrete probability measure $\nu_{\alpha \beta} \in \mu_{\alpha}+N_{\beta}$. Let $r(\mu)=x, r\left(\mu_{\alpha}\right)=x_{\alpha}$ and $r\left(\nu_{\alpha \beta}\right)=x_{\alpha \beta}$. For each $\alpha, \nu_{\alpha \beta}$ converges to $\mu_{\alpha}$, so by Proposition 5, $x_{\alpha \beta}$ converges to $x_{\alpha}$. Now the product set of all pairs $(\alpha, \beta)$, with the product order, is directed and the net $\left\{\nu_{\alpha \beta}\right\}$ converges along this ordered set to $\mu$. By Proposition $5,\left\{x_{\alpha \beta}\right\}$ converges to $x$. It follows that any closed neighborhood of $x$ eventually contains all $x_{\alpha \beta}$, hence eventually contains all $x_{\alpha}$. Thus $\left\{x_{\alpha}\right\}$ converges to $x$.

It remains to show that the existence of a barycentre map allows us to embed $X$ in a locally convex topological vector space. This follows from a general result (Proposition 7 below) about compact sets which says that if $X$ is a compact Hausdorff space with a "barycentre" map, then we can introduce a convex structure with lots of continuous affine functions and the given map as its resultant map.

Proposition 7. Suppose $X$ is a compact, Hausdorff space and $r: P(X) \rightarrow X$ has the following properties:

(i) $\quad r$ is continuous,

(ii) $r\left(\delta_{x}\right)=x$ for all $x \in X$,

(iii) if $\mu, \nu \in P(X)$ and $\omega=\alpha \mu+\beta \nu$ for $\alpha, \beta>0, \alpha+\beta=1$ then any one of the elements $r(\mu), r(\nu)$ and $r(\omega)$ is determined by the other two. Then there is a continuous injection $\phi: X \rightarrow F$ which maps $X$ onto a convex subset $Y$ of a locally convex TVS $F$, such that if we denote by $\bar{r}$ the resultant map for $Y$ then for any $\mu \in P(X), \bar{r}\left(\mu \cdot \phi^{-1}\right)=\phi(r(\mu))$.

Proof. Define

$$
A=\{f \in C(X): \mu(f)=f(r(\mu)) \text { for all } \mu \in P(X)\} .
$$

Then $A$ is a uniformly closed subspace of $C(X)$ which contains the constants. Our first task will be to show that $A$ separates points of $X$. If we let

$$
K=\{\mu-\nu: \mu, \nu \in P(X), r(\mu)=r(\nu)\},
$$

then $K$ is weak ${ }^{*}$ compact since it is a continuous image of

$$
Q=\{(\mu, \nu) \in P(X) \times P(X): r(\mu)=r(\nu)\}
$$

which is compact by (i). Also $K$ is symmetric and convex. Indeed if $\mu-\nu \in K$ and $\mu^{\prime}-\nu^{\prime} \in K$ and $\alpha, \beta>0, \alpha+\beta=1$, then $r(\mu)=r(\nu)$, $r\left(\mu^{\prime}\right)=r\left(\nu^{\prime}\right)$ and $\alpha(\mu-\nu)+\beta\left(\mu^{\prime}-\nu^{\prime}\right)=\left(\alpha \mu+\beta \mu^{\prime}\right)-\left(\alpha \nu+\beta \nu^{\prime}\right)$ which is in $K$ by (iii). 
If $L=\bigcup_{n=1}^{\infty} n K$, then $L$ a linear subspace of $M(X)$ since $K$ is radial. We shall show that $L$ is weak* closed. By the Banach-Krein-Smulyan Theorem, it is enough to show that $L \cap B_{2}$ is weak* compact where $B_{2}=\{\lambda \in M(X):\|\lambda\| \leqq 2\}$. We will show, in fact, that $L \cap B_{2}=K$. Take $\lambda=n(\mu-\nu) \in L \cap B_{2}$ and suppose $\|\lambda\|=2$. (The case $\|\lambda\|<2$ will follow since $K$ is radial.) Let $\bar{\mu}=\lambda^{+}$and $\bar{\nu}=\lambda^{-}$. Since $\|\bar{\mu}\|+\|\bar{\nu}\|=\|\lambda\|=2$ and $\|\bar{\mu}\|-\|\bar{\nu}\|=\bar{\mu}(1)-\bar{\nu}(1)=\lambda(1)=0$, it follows that $\bar{\mu}, \bar{\nu} \in P(X)$. Denote $\omega=n(\mu \wedge \nu) /(n-1)$. (If $n=1$, we are finished.) Then $\omega \geqq 0$ and

$$
\mu=(\mu-\nu)^{+}+(\mu \wedge \nu)=(\bar{\mu} / n)+(n-1) \omega / n .
$$

Similarly $\nu=(\bar{\nu} / n)+(n-1) \omega / n$. Since these are affine combinations, $\omega \in P(X)$ and since $r(\mu)=r(\nu)$, it follows from (iii) that $r(\bar{\mu})=$ $r(\bar{\nu})$. Hence $\lambda=\bar{\mu}-\bar{\nu} \in K$. We have shown that $L$ is weak* closed.

Now to show that $A$ separates points of $X$, take two distinct points $x, y$ in $X$. Then by (ii) $r\left(\delta_{x}\right) \neq r\left(\delta_{y}\right)$, hence $\delta_{x}-\delta_{y} \notin K=L \cap B_{2}$. Since $\left\|\delta_{x}-\delta_{y}\right\|=2$, it follows that $\delta_{x}-\delta_{y} \notin L$. Since $L$ is weak* closed we can use the Hahn-Banach Theorem to construct a weak* continuous linear functional $F$ on $M(X)$ such that $F\left(\delta_{x}-\delta_{y}\right)>F(L)$. Since $L$ is a subspace, $F(L)=0$, and $F\left(\delta_{x}\right)>F\left(\delta_{y}\right)$. If we let $f=F \circ \delta$ (where $\delta: x \rightarrow \delta_{x}$ is the natural homeomorphism), then $f \in C(X)$ and $f(x)=$ $F\left(\delta_{x}\right)>F\left(\delta_{y}\right)=f(y)$. To show that $f \in A$, observe that for any $\mu \in$ $P(X), \mu-\delta_{r(\mu)} \in K$ by (ii), hence $F\left(\mu-\delta_{r(\mu)}\right)=0$ and so

$$
\mu(f)=F(\mu)=F\left(\delta_{r(\mu)}\right)=f(r(\mu)) .
$$

Since $A$ separates points of $X$, the natural map $\phi: X \rightarrow A^{*}$ is an injection. If we give $A *$ the weak* topology (which is locally convex), then $\phi$ is continuous (since members of $A$ are continuous on $X$ ). Since

$$
\alpha \phi(x)+\beta \phi(y)=\phi\left(r\left(\alpha \delta_{x}+\beta \delta_{y}\right)\right)
$$

(evaluate each side on any $f$ in $A$ ), we deduce that $\phi(X)$ is convex. Now let $\bar{r}$ denote the resultant map in $\phi(X)$ and for any $f$ in $A$ let $\bar{f}$ denote the weak* continuous linear functional obtained when $f$ acts on $A^{*}$. Then if $\mu \in P(X)$,

$$
\bar{f}\left(\bar{r}\left(\mu \cdot \phi^{-1}\right)\right)=\left(\mu \cdot \phi^{-1}\right)(\bar{f})=\mu(f)=f(r(\mu))=\bar{f}(\phi(r(\mu))) .
$$

Proposition 7 is quite interesting. There is a category-theoretic version of the theorem which was first proved by Swirszcz [8] using the machinery of category theory. In categorical language it says that the category Compconv is algebraic over the category Comp. These ideas 
are laid out in [7] where essentially our proof of Proposition 7 is presented [7, Prop. 7.3].

Now we will prove the main theorem.

THEOREM 1. Suppose $X$ is a compact convex set which is strongly locally convex. Then there is an affine homeomorphism mapping $X$ onto a convex subset of a locally convex topological vector space.

Proof. If $X$ is strongly locally convex, then by Proposition 6, there is a barycentre map $r$ for $X$. Such a map clearly satisfies (i), (ii) and (iii) of Proposition 7 ((iii) since $r$ is affine), and so there is a continuous injection $\phi$ of $X$ onto a convex subset $Y$ of a locally convex topological vector space. The condition $\bar{r}\left(\mu \cdot \phi^{-1}\right)=\phi(r(\mu))$ applied to measures which live on two points tells us that $\phi$ is affine.

4. The locally compact case. It is not difficult to extend the main embedding theorem proved in the preceding section to locally compact convex sets. The argument proceeds from the following simple continuity criterion for affine functions.

Lemma. Suppose $X$ is a convex set and $F: X \rightarrow \mathbf{R}$ an affine function. Suppose $x, p$ and $y$ are distinct points of $X$ with $p$ on the line segment joining $x$ and $y$. If $f$ is continuous at $p$, then $f$ is continuous at $x$.

Proof. Suppose $x_{\gamma} \rightarrow x$. By hypothesis on $p$, there is some $\lambda$ in $(0,1)$ with $p=\lambda x+(1-\lambda) y$. Since the topology is linear, the net $p_{\gamma}=\lambda x_{\gamma}+(1-\lambda) y$ converges to $p$. Since $f$ is affine and continuous at $p$, $\lambda f\left(x_{\gamma}\right)+(1-\lambda) f(y)=f\left(p_{\gamma}\right)$ converges to $f(p)=\lambda f(x)+(1-\lambda) f(y)$. This is a statement about convergence of real numbers, so cancelling $(1-\lambda) f(y)$ and then dividing by $\lambda \neq 0$, we get $f\left(x_{\gamma}\right) \rightarrow f(x)$.

THEOREM 2. Suppose $X$ is a locally compact convex set which is strongly locally convex. Then there is an affine homeomorphism mapping $X$ onto a convex subset of a locally convex TVS.

Proof. Let $K$ be a compact convex neighborhood of a point $x$ in $X$. Then any $f^{\prime}$ in $A(K)$ can be extended algebraically to an affine function $f$ on all of $X$ since $X$ is contained in the affine span of $K$. Moreover by the lemma since $f$ is continuous on $K$, it will be continuous on all of $X$. Thus there is a one-to-one correspondence between $A(K)$ and the affine continuous functions on $X$, denoted $A(X)$. By Theorem 1 , since $K$ is strongly locally convex, $A(K)$ separates the points of $K$. Hence $A(X)$ separates the points of $X$. 
Let $A(X)^{*}$ denote the algebraic dual of $A(X)$ with the weak*, i.e. $\sigma\left(A(X)^{*}, A(X)\right)$, topology. Then $A(X)^{*}$ is a locally convex topological vector space. Since $A(X)$ separates the points of $X$, the map $x \mapsto(f \mapsto f(x))$ is an affine one-to-one embedding of $X$ into $A(X)^{*}$. It only remains to show that the weak* topology restricted to $X$ is the same as the original topology on $X$.

First if $\left\{x_{\alpha}\right\}$ converges to $x$ in $X$ with the original topology, then $\left\{f\left(x_{\alpha}\right)\right\}$ converges to $f(x)$ for all $f$ in $A(X)$ and so $\left\{x_{\alpha}\right\}$ converges to $x$ in the weak ${ }^{*}$ topology restricted to $X$. On the other hand, suppose $\left\{x_{\alpha}\right\}$ converges to $x$ in the weak* topology but not in the original topology. Then there is a compact neighborhood $U$ of $x$ in the original topology and a subnet $\left\{x_{\beta}\right\}$ of $\left\{x_{\alpha}\right\}$ such that $x_{\beta} \notin U$ for all $\beta$. For each $\beta$ choose $\lambda_{\beta}$ in $(0,1)$ such that $y_{\beta}=\lambda_{\beta} x_{\beta}+\left(1-\lambda_{\beta}\right) x$ is in $U \backslash$ int $U$. Since in the original topology $U \backslash$ int $U$ is compact, $\left\{y_{\beta}\right\}$ has a convergent subnet $\left\{y_{\gamma}\right\}$ converging to $y \in U \backslash$ int $U$. Thus from above $\left\{y_{\gamma}\right\}$ converges to $y$ in the weak* topology, and since in the weak* topology $\left\{x_{\beta}\right\}$ converges to $x$, we deduce $\left\{y_{\gamma}\right\}$ converges to $x$ also. But $y \in U \backslash$ int $U$, so that $y$ is distinct from $x$. This contradiction forces the topologies to be identical and the proof is complete.

5. We say that a convex set $X$ is weakly locally convex if every $x$ in $X$ has a neighborhood basis of convex sets. This is formally weaker than strong local convexity (where we require the neighborhoods to be open) but it is not known whether the two notions coincide. To emphasize the formal difference between them, let us remark that it is not generally true that the interior of a convex subset of $X$ is convex. In fact one has the following proposition.

Proposition 8. Suppose $X$ is a compact convex set which is weakly locally convex. If the set of extreme points of $X$ is not closed in $X$, then there exists a convex subset $K$ of $X$ whose interior is not convex.

Proof. Suppose $\lim x_{\alpha}=x$, where $x_{\alpha}$ are extreme but $x \in X$ is not. Then $x=y / 2+z / 2$ for some distinct $y, z \in X$. Choose disjoint closed convex neighborhoods $U$ of $y$ and $V$ of $z$ such that $x \notin U U$ $V$. Then $K=\operatorname{conv}(U \cup V)$ is a convex subset of $X$ containing $x$. Certainly $y$ and $z$ are in the interior of $K$, but since $x_{\alpha} \notin U \cup V$ for sufficiently large $\alpha$ (since $U \cup V$ is closed and $x \notin U \cup V$ ), $x_{\alpha}$ is eventually outside $K$ (since $x_{\alpha}$ extreme), and hence $x$ is not in the interior of $K$.

As is well-known, there exist examples, even in $\mathbf{R}^{3}$, of compact convex sets whose extreme points are not closed. In infinite dimensional spaces the situation is even more striking since compact convex sets exist whose extreme points are dense. In fact, if we regard the 
compact convex subsets of an infinite dimensional Banach space as points in a complete metric space under the Hausdorff metric, then the results of Klee [4] show that the set of compact convex sets whose extreme points are not dense is of the first Baire category.

There are some partial results which suggest that strong and weak local convexity may be equivalent for compact convex subsets of a topological vector space. Let us extend the notion of local convexity of a compact convex set $X$ by saying that a closed convex subset $K$ of $X$ is regular if every open set containing $K$ contains a convex neighborhood of $K$. Thus, $X$ is weakly locally convex if each point of $X$ is regular. If every closed convex subset of $X$ is regular, then it can be shown that $X$ is strongly locally convex. Also if $X$ is a compact convex subset of a TVS, and the relative topology on $X$ is weakly locally convex, then it is known that every finite dimensional closed convex subset of $X$ is regular.

Another result is that if $X$ is a compact convex subset of a topological vector space and the compact convex set $X-X$ is weakly locally convex at 0 , then $X$ is strongly locally convex. This result together with the Baire category theorem yields the following. (Recall that a point $x$ is an internal point of $X$ if $x$ is in the one-dimensional interior of $L \cap X$ for every line $L$ contained in the affine span of $X$ and passing through $x$.) If $X$ is a weakly locally convex compact convex set in a topological vector space and if $X$ has an internal point, then $X$ is strongly locally convex.

The outstanding open problem in this area is the question of whether every compact topological convex set can be embedded in a locally convex topological vector space. ${ }^{2}$ Of course, if one were to find a compact convex set which was weakly but not strongly locally convex, then one would have a counterexample for this open problem. But we prefer to believe that weak and strong local convexity coincide.*

If one defines a topological convex set as a convex set $X$ of a real linear space endowed with a Hausdorff topology such that the map $(x, y, t) \mapsto t x+(1-t) y$ is continuous from $X \times X \times[0,1]$ into $X$, then all of the results of this paper are true for (locally) compact topological convex sets. This setting is really no more general, however, since it follows from Theorem 3.2 of [5] that a locally compact topological convex set can be embedded by an affine homeomorphism into a topological vector space. The unpublished dissertation of S. Dubuc [1] contains a development of general topological convex sets as well as a construction of affine functions similar to our discussion in $\$ 2$. He also gives a

${ }^{2}$ It appeared recently in the Notices.

* These notions do, in fact, coincide. J. W. Roberts has recently shown, using the barycentre approach of Prop. 7, that Theorem 1 is true with weak local convexity. His paper will appear in the Canadian Journal of Mathematics. 
concise treatment of the algebraic structure of convex sets as well as a proof of the expected theorem on embedding abstract convex sets into linear spaces.

\section{REFERENCES}

1. S. Dubuc, Topological Convex Sets, unpublished dissertation, Cornell University, Ithaca, New York, 1966.

2. John Kelley, General Topology, Princeton, Van Nostrand, 1955.

3. J. L. Kelly, I. Namioka, et al., Linear Topological Spaces, Princeton, Van Nostrand, 1963.

4. V. L. Klee, Some new results on smoothness and rotundity in normed linear spaces, Math. Annalen 139: (1959), 51-63.

5. J. Lawson and B. Madison, On congruences and cones, Math. Z., 120: (1971), 18-24.

6. R. R. Phelps, Lecture on Choquet's Theorem, Princeton, Van Nostrand Mathematical Studies \#7, Van Nostrand, 1966.

7. Z. Semadeni, Monads and Their Eilenberg-Moore Algebras in Functional Analysis, Queen's Papers in Pure and Applied Mathematics, No. 33. Queen's University, Kingston, Ontario, Canada, 1973.

8. T. Swirszcz, Monadic functors in convexity, Bull. Acad. Polon. Sci., 22 (1974), 39-42.

Received June 19, 1975 and in revised form August 29, 1975.

QueEn's University, CANADA 




\section{Pacific Journal of Mathematics}

Vol. 64, No. 1

May, 1976

Walter Allegretto, Nonoscillation theory of elliptic equations of order $2 n \ldots \ldots \quad 1$

Bruce Allem Anderson, Sequencings and starters.................. 17

Friedrich-Wilhelm Bauer, A shape theory with singular homology .......... 25

John Kelly Beem, Characterizing Finsler spaces which are

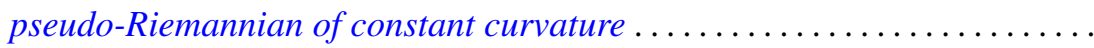

Dennis K. Burke and Ernest A. Michael, On certain point-countable

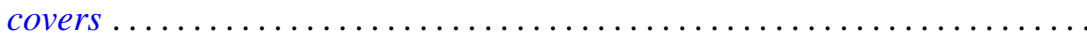

Robert Chen, A generalization of a theorem of Chacon ............... 93

Francis H. Clarke, On the inverse function theorem ................ 97

James Bryan Collier, The dual of a space with the Radon-Nikodým

property ....................................... 103

John E. Cruthirds, Infinite Galois theory for commutative rings ............ 107

Artatrana Dash, Joint essential spectra......................... 119

Robert M. DeVos, Subsequences and rearrangements of sequences in FK

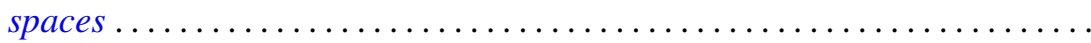

Geoffrey Fox and Pedro Morales, Non-Hausdorff multifunction generalization

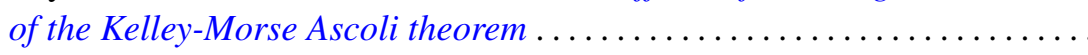

Richard Joseph Fleming, Jerome A. Goldstein and James E. Jamison, One

parameter groups of isometries on certain Banach spaces.............

Robert David Gulliver, II, Finiteness of the ramified set for branched

immersions of surfaces

Kenneth Hardy and István Juhász, Normality and the weak cb property ...... 167

C. A. Hayes, Derivation of the integrals of $L^{(q)}$-functions.

Frederic Timothy Howard, Roots of the Euler polynomials .

Robert Edward Jamison, II, Richard O'Brien and Peter Drummond Taylor, On

embedding a compact convex set into a locally convex topological vector space ....................................

Andrew Lelek, An example of a simple triod with surjective span smaller than span ...

Janet E. Mills, Certain congruences on orthodox semigroups

Donald J. Newman and A. R. Reddy, Rational approximation of $e^{-x}$ on the positive real axis.

John Robert Quine, Jr., Homotopies and intersection sequences ...

Nambury Sitarama Raju, Periodic Jacobi-Perron algorithms and fundamental units ....

Herbert Silverman, Convexity theorems for subclasses of univalent functions. . .

Charles Frederick Wells, Centralizers of transitive semigroup actions and endomorphisms of trees.........................

Volker Wrobel, Spectral approximation theorems in locally convex spaces ..................... 